\title{
Managing Pediatric Cancer Patients in COVID19 Pandemic
}

\author{
Vikas Garg $^{1}$ - Sameer Bakhshi ${ }^{1}$ - Gopila Gupta ${ }^{2}$. Deepam Pushpam ${ }^{1}$
}

Received: 15 April 2020 / Accepted: 4 May 2020 / Published online: 15 May 2020

(C) Dr. K C Chaudhuri Foundation 2020

To the Editor: Data of COVID19 in pediatric cancer is still emerging. In immunosuppressed children human coronavirus infections are more severe [1], but the same may not hold true for COVID19. A survey identified nine patients with pediatric cancer and COVID19; all had mild symptoms and clinical course [2]. In Italy, oncology services are being continued despite being severely affected by COVID19 [3]. In India, lockdown was enforced to curb the spread of COVID which restricted patient movement in hospitals. Our tertiary care center was designated as COVID19 hospital with beds and health care workers (HCW) rationed for COVID19. There were interruptions in the already curtailed services when occasional HCW developed COVID19 [4]. From community perspective, pediatric patients may act as asymptomatic carriers leading to community spread due to overcrowding and generations living together.

To manage these unprecedented problems, a consensus was arrived amongst the team looking after pediatric cancers regarding optimal management, considering our resources and logistics. We classified patients into three groups, A- easy access to our center; B- difficult access and C- inability to come due to lockdown. Group A patients were treated as per their ongoing protocol. For group B patients, the frequency of chemotherapy cycles was increased, weekly chemotherapy doses were omitted, dose reductions were considered for severely myelotoxic therapies, and nearby home administration of subcutaneous drugs were encouraged. Group C patients were offered oral therapies [5]. Patients who were in maintenance phase of acute lymphoblastic leukemia, receiving tyrosine kinase inhibitors for chronic myeloid leukemia and survivors were managed telephonically instead of hospital visits.

Deepam Pushpam

deepampushpam@gmail.com

1 Department of Medical Oncology, Dr. BRA IRCH, All India Institute of Medical Sciences (AIIMS), New Delhi, India

2 Department of Hematology, All India Institute of Medical Sciences (AIIMS), New Delhi, India
For highly curable and aggressive malignancies with potential for early mortality like Non-Hodgkin' lymphoma and acute leukemia, an effort was made to mobilize social support services to ensure patients' access to oncology services. We are reviewing our treatment plans regularly to incorporate evolving data. However, we feel that Italian approach which emphasizes on nasopharyngeal swab testing of patient and caregivers prior to admission, N95 masks to patients and personal protective equipment for patients and caregivers might not be feasible in most parts of our country [3]. We need institute specific protocols depending on the type of patients being catered, whether they are designated for COVID care or not, so as to balance good oncology related outcome with prevention of community spread of COVID.

\section{Compliance with Ethical Standards}

Conflict of Interest None.

\section{References}

1. Ogimi C, Englund JA, Bradford MC, Qin X, Boeckh M, Waghmare A. Characteristics and outcomes of coronavirus infection in children: the role of viral factors and an immunocompromised state. J Pediatr Infect Dis Soc. 2019;8:21-8.

2. Hrusak O, Kalina T, Wolf J, et al. Flash survey on severe acute respiratory syndrome coronavirus- 2 infections in paediatric patients on anti-cancer treatment. Eur J Cancer. 2020 Apr 7;132:11-6. https:// doi.org/10.1016/j.ejca.2020.03.021.

3. Sainati L, Biffi A. How we deal with the COVID-19 epidemic in an Italian pediatric onco-hematology clinic located in a region at high density of cases. Br J Haematol. 2020 Apr 16; https://doi.org/10. 1111/bjh.16699.

4. AIIMS Nurse, Guard Test Positive, Cancer Patients Advised Quarantine. Business Insider. Available at: https://www. businessinsider.in/india/news/security-guard-posted-at-healthministers-osd-office-nurse-test-positive-for-covid-19-in-aiims/ articleshow/75396138.cms. Accessed 27 Apr 2020.

5. Pramanik R, Bakhshi S. Metronomic therapy in pediatric oncology: a snapshot. Pediatr Blood Cancer. 2019;66:e27811.

Publisher's Note Springer Nature remains neutral with regard to jurisdictional claims in published maps and institutional affiliations. 